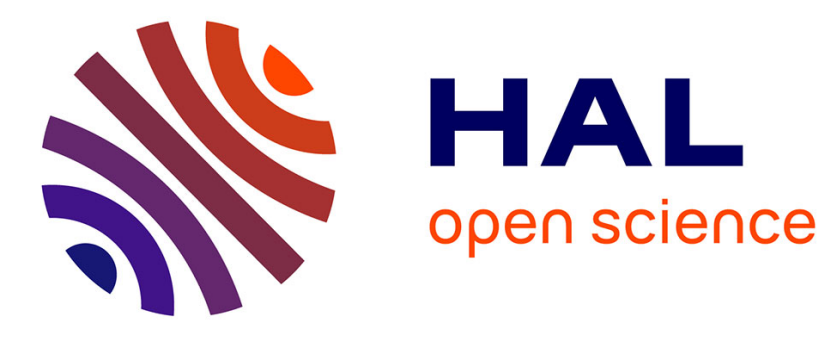

\title{
Particle-Size-Exclusion Clogging Regimes in Porous Media
}

Gaetan Gerber, Stéphane Rodts, Patrick Aimedieu, Pamela Françoise Faure, Philippe Coussot

\section{To cite this version:}

Gaetan Gerber, Stéphane Rodts, Patrick Aimedieu, Pamela Françoise Faure, Philippe Coussot. Particle-Size-Exclusion Clogging Regimes in Porous Media. Physical Review Letters, 2018, 120 (14), 10.1103/PhysRevLett.120.148001 . hal-02912560

\section{HAL Id: hal-02912560 \\ https://hal-enpc.archives-ouvertes.fr/hal-02912560}

Submitted on 6 Aug 2020

HAL is a multi-disciplinary open access archive for the deposit and dissemination of scientific research documents, whether they are published or not. The documents may come from teaching and research institutions in France or abroad, or from public or private research centers.
L'archive ouverte pluridisciplinaire HAL, est destinée au dépôt et à la diffusion de documents scientifiques de niveau recherche, publiés ou non, émanant des établissements d'enseignement et de recherche français ou étrangers, des laboratoires publics ou privés. 


\title{
Particle-Size-Exclusion Clogging Regimes in Porous Media
}

\author{
G. Gerber ${ }^{1,2}$ S. Rodts, ${ }^{1}$ P. Aimedieu, ${ }^{1}$ P. Faure, ${ }^{1}$ and P. Coussot ${ }^{1}$ \\ ${ }^{1}$ Université Paris-Est, Laboratoire Navier (ENPC-IFSTTAR-CNRS), Champs sur Marne, France \\ ${ }^{2}$ Experimental Soft Condensed Matter Group, School of Engineering and Applied Sciences, \\ Harvard University, Cambridge, Massachusetts, USA
}

(Received 26 September 2017)

\begin{abstract}
From observations of the progressive deposition of noncolloidal particles by geometrical exclusion effects inside a 3D model porous medium, we get a complete dynamic view of particle deposits over a full range of regimes from transport over a long distance to clogging and caking. We show that clogging essentially occurs in the form of an accumulation of elements in pore size clusters, which ultimately constitute regions avoided by the flow. The clusters are dispersed in the medium, and their concentration (number per volume) decreases with the distance from the entrance; caking is associated with the final stage of this effect (for a critical cluster concentration at the entrance). A simple probabilistic model, taking into account the impact of clogging on particle transport, allows us to quantitatively predict all these trends up to a large cluster concentration, based on a single parameter: the clogging probability, which is a function of the confinement ratio. This opens the route towards a unification of the different fields of particle transport, clogging, caking, and filtration.
\end{abstract}

DOI:

A multitude of situations involve the passage or stoppage of elements suspended in a liquid through a porous medium: separation of species in chromatography or microfluidics [1], filtration in biological processes [2], water and wastewater treatment [3], drilling and oil recuperation processes [4], pollutant transport or storage in soils [5], and sediment transport [6]. Depending on the characteristics of the elements or of the porous medium, different situations may be encountered: The elements may be transported without being stopped, be transported at some depth and finally stopped - this is depth filtration - or be blocked at the entrance-this is caking (outside the filter). Various crucial questions of practical importance emerge: the efficiency of the filter (generally a porous medium), conditions leading to caking, depth reached by stopped elements, induced evolution of flow characteristics and filtration properties, etc. So far, theories or experiments essentially focused separately on caking [7], clogging at the entrance of a filter or a model $2 \mathrm{D}$ porous medium [8], deep-bed filtration [9], permeability evolutions [10], or colloid transport [11], and recent studies showed the possibility of observing indirectly [12] or directly [13] particle depositions in depth in porous media. These studies provided insights into the physical mechanisms of these different processes under specific conditions, but there is no unifying physical approach allowing us to deal simply, at first order, with the different regimes (transport, depth filtration, and caking).

Here we focus on the case for which, whatever the different possible effects at work (Brownian motion, colloidal interactions with walls, sedimentation, aggregation between elements, drag force due to flow, etc.), the porous medium clogs after a more or less long time of flow. In that aim, we use a model system for which clogging can result only from size exclusion (particle jamming in a path smaller than its diameter). We show that clogging occurs as an accumulation of elements in pore size clusters, which ultimately constitute regions avoided by the flow. The clusters are dispersed in the medium, and their concentration (number per volume) decreases with the distance from the entrance, a process ultimately leading to caking. A model involving a single parameter, i.e., the probability to form a cluster, but taking into account the impact of the existence of clogged paths, allows us to predict all the trends observed inside the sample. Systems with additional, more complex interactions between the elements and the wall might be considered within a similar frame, through a probability of blockage (or attachment) now depending also on these effects.

Our experiment consists in making a dilute suspension of noncolloidal particles flow through a model porous medium and then following the clogging dynamics. The particles are monodisperse, spherical, polystyrene beads with a diameter of $41 \pm 9 \mu \mathrm{m}$. The carrying fluid is a glycerol-water mixture chosen to match the density of the particles and avoid sedimentation effects (see [14]). The flow is imposed at a constant flow rate, and all our tests are carried out under laminar flow conditions (maximum Reynolds number at the pore scale $<1$ ). The porous medium is composed of almost monodisperse spherical glass grains stacked at random close packing in a glass tube of a diameter much larger than that of the grains $\left(d_{g}\right)$. Various particle to grain ratios $(r)$ are tested in the range [0.054-0.13], which corresponds to the range for which 
deposits are expected [15]. In the absence of significant physical effects varying with the grain or particle size, filtration properties are expected to depend solely on $r$.

In order to measure the distribution of particle concentration in the sample, a benchtop NMR spectrometer is used with an additional gradient field over the vertical axis (i.e., that of the glass tube), which makes it possible to perform proton density unidirectional profiles [14]. The measured NMR signal corresponds only to the protons of the liquid phase, from which we deduce the local solid fraction $\left(\Phi_{S}\right)$ in each cross section. NMR measurements are carried out after successive periods of time, corresponding to a constant additional number of injected particles. After each of these periods, we maintain the flow with liquid only, so as to remove unblocked particles, and then a 1D profile imaging starts. The corresponding successive profiles along the depth $(x)$ axis (in grain diameter unit) thus provide a clear view of the clogging dynamics.

Two mechanisms appear: (1) in-depth deposition, where particles are clogged at various depths in the medium, inducing a local increase of $\Phi_{S}$; and (2) surface deposition or caking, where particles tend to accumulate at the surface and create a packing of particles on top of the sample. Different clogging regimes are observed when increasing $r$. For $r<0.05$, no particle clogged in the porous medium is detected by our technique. For a slightly larger $r$ value, only a slight in-depth deposition is visible after our series of injections [see Fig. 1(a)]: The height of the profiles increases slowly close to the sample top. For larger $r$ [e.g., 0.11 in Fig. 1(b)], in-depth deposition becomes significant, and, beyond a critical number of injected particles, surface deposition begins. However, at the same time, for increasing $r$, the in-depth deposition tends to be more concentrated close to the top surface, and surface deposition begins earlier, i.e., for a smaller number of injected particles [e.g., $r=0.13$ in Fig. 1(c)]. At the beginning of surface deposition, there may still be some residual in-depth deposition due to some unevenness of the process, but soon this effect completely disappears [see Fig. 1(c)]: A few-particle-high cake forms, which obviously does not allow the passage of other particles. The solid fraction in this cake is $52 \%$, which is consistent with a loose nonvibrated random packing [16].

For a better appreciation of the physical situation, the deposited solid volume may be represented in terms of the density $(n)$ of blocked particles, i.e., the number $N_{b}$ of blocked particles in a slice of elementary thickness $e$ divided by the (maximum) number $N$ of particles in this slice at a concentration in the pore space equal to that in the cake. Considering the pore structure, we can reasonably take $e=d_{g} / 2$. Then we see that, during a first stage, the particles are regularly deposited in depth (see Fig. 2). This process goes on up to a critical density $(n=45 \pm 5 \%)$ significantly smaller than the maximum one (i.e., $n=1$ ), for which the particles start to be significantly deposited above the sample surface [see Figs. 1(b), 1(c), and 2]. This cake growth coincides with the end of the in-depth deposition (see Fig. 2), which provides a clear criterion for a cake beginning (no particle can be transported through the particle-packed cake). Furthermore, during the in-depth deposition regime, for each depth, $n$ increases linearly with time: We get a master curve when rescaling $N_{b}$ by the number $N_{i}$ of injected particles since the beginning of the test (see the inset in Fig. 2). Thus, during the first injections, in-depth deposition is predominant and the evolution of the particle distribution in depth remains unchanged (it varies linearly with $N_{i}$ ). This unexpected result means that the particles go on discovering and populating new sites regardless of the particles already blocked, even if they occupy up to about $50 \%$ of the available volume. Then the situation suddenly changes: The in-depth deposition becomes negligible, and caking starts, as if some percolation network formed, precluding a further penetration of particles in the sample.
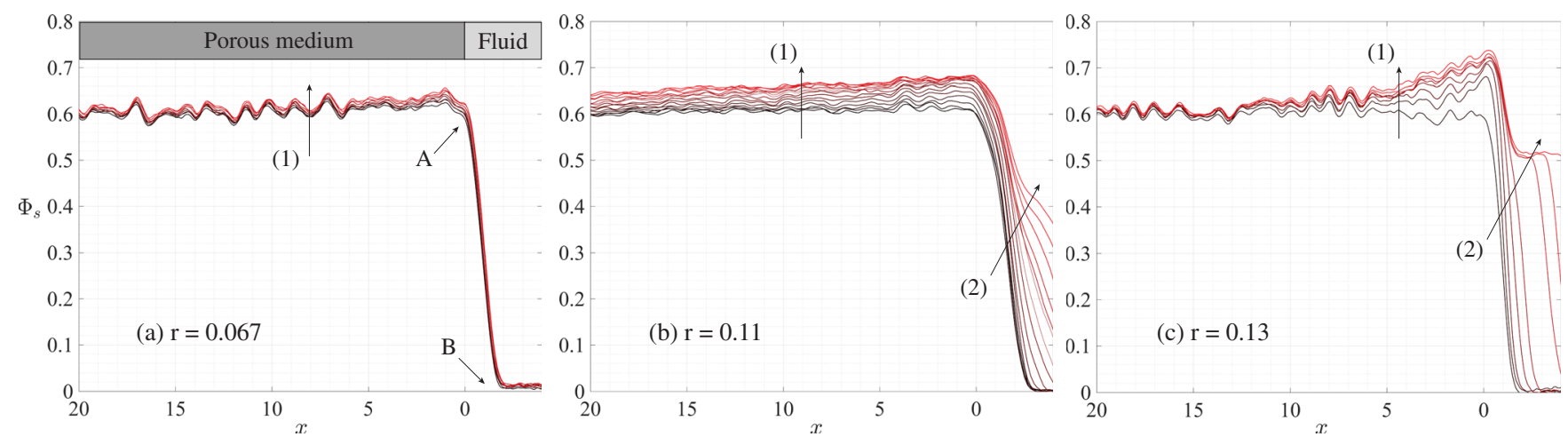

F1:1 FIG. 1. Successive injections of the same suspended particle number $\left(5.0 \times 10^{5}\right)$ for different $r$ values through bead packing: solid F1:2 volume concentration profile after each injection (solid lines, from bottom to top). The lowest curve (darkest) accounts for the initial state, where $\Phi_{S} \approx 60 \%$ in the medium. The oscillations around this value result from a specific local arrangement of grains, which is stable, as proved by the stability of these oscillations during flow. Note that, due to the nonperfect flatness of the free surface, the initial profile collapses to zero at the sample top, with some slope (from $A$ to $B$ ). 


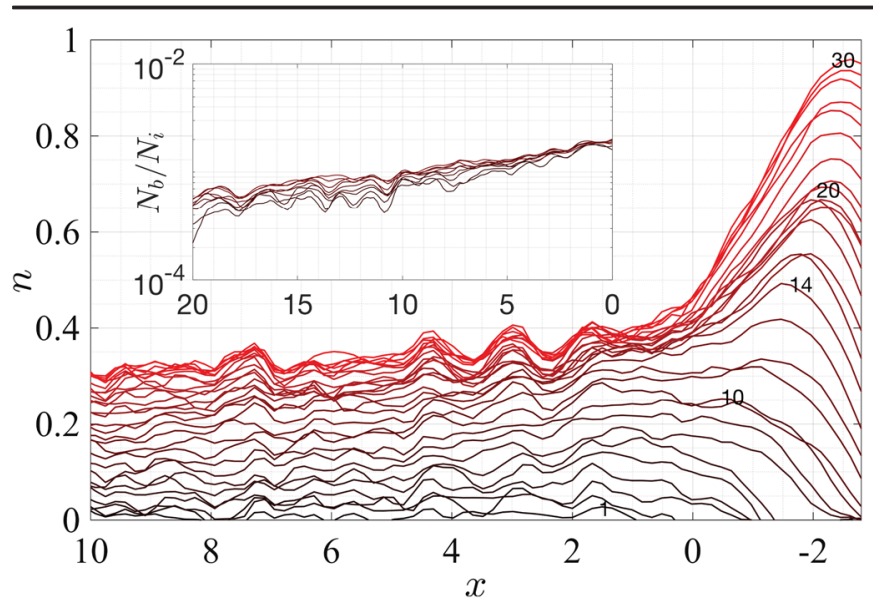

FIG. 2. Successive (numbered from bottom to top) injections of the same suspended particle number for $r=0.11$ density vs depth. Note that there is some apparent accumulation of particles just above the free surface, which is due to the nonperfect flatness of the sample (see Fig. 1). The inset shows the blocked to injected particle ratio for injections $1-14$.

In order to understand these trends, we directly look at the distribution of particles at the local scale by x-ray $\mu$ tomography, performed after a series of injections (see Fig. 3). This shows an original arrangement of the particles: They are essentially distributed in clusters, whose apparent volume $\left(\propto d_{c}^{3}\right)$ contains from a few tens to a few hundreds of particles, plus some isolated particles (i.e., $d_{c} / d_{p} \approx 1$ ) [see Fig. 3(b)]. Surprisingly, this observation is valid even when these clusters and the isolated particles occupy a small fraction of void in the porous medium [see Fig. 3(a)]. That means that during all the filtration process the particles are preferentially blocked in dispersed clusters. These characteristics of cluster distribution are valid even around the critical concentration for a transition to caking [see Fig. 3(c)]: The particles are still distributed in large clusters separated by void regions, but now the clusters seem connected.
These observations allow us to deduce key elements concerning the process of particle deposition and cluster formation. The local width of pathways experienced by elements generally ranges from zero to a maximum value $d$, so that blockage is a matter of probability: An element of a size smaller than $d$ will be blocked if it is draught by the liquid through a sufficiently narrow area. Here, since the clusters are essentially independent, have a size of the order of the voids between neighboring grains, and include almost all the clogged particles, the variations of $n$ in time may be considered to be essentially due to the formation of new clusters. They likely nucleate from some initial local clogging of one particle and then grow more rapidly than our time resolution (i.e., changes between two successive injections).

Moreover, the first injected particles partially block the most probable intergrain sites, but those events likely do not significantly affect the flow through the porous medium. Other particles can soon follow the same paths and arrive ahead of the blocked particles with a probability of blocking close to unity. This process goes on until the cluster size is sufficient to affect the flow (loss of permeability) at the pore scale, typically by diverting most of the flux towards other pores, which also explains that the cluster size then stops growing, as it has reached a size of the order of the pore size.

On this basis, we can build a simple model to describe the evolution of the number of blocked particles as a function of the depth and the number of injected particles $N_{i}$. We represent the porous medium as successive identical layers of thickness $e$, whose value (i.e., $d_{g} / 2$ ) appears consistent with the process of dispersed clusters filling pores. Because of the percolation effect above described, the maximum achievable number of particles in a given layer during the experiment is not $N$ but a fraction of this number $(\approx 0.45 N)$. The particles are dispersed homogenously at random in the flowing liquid and can reach various positions in a layer, possibly leading to clogging.
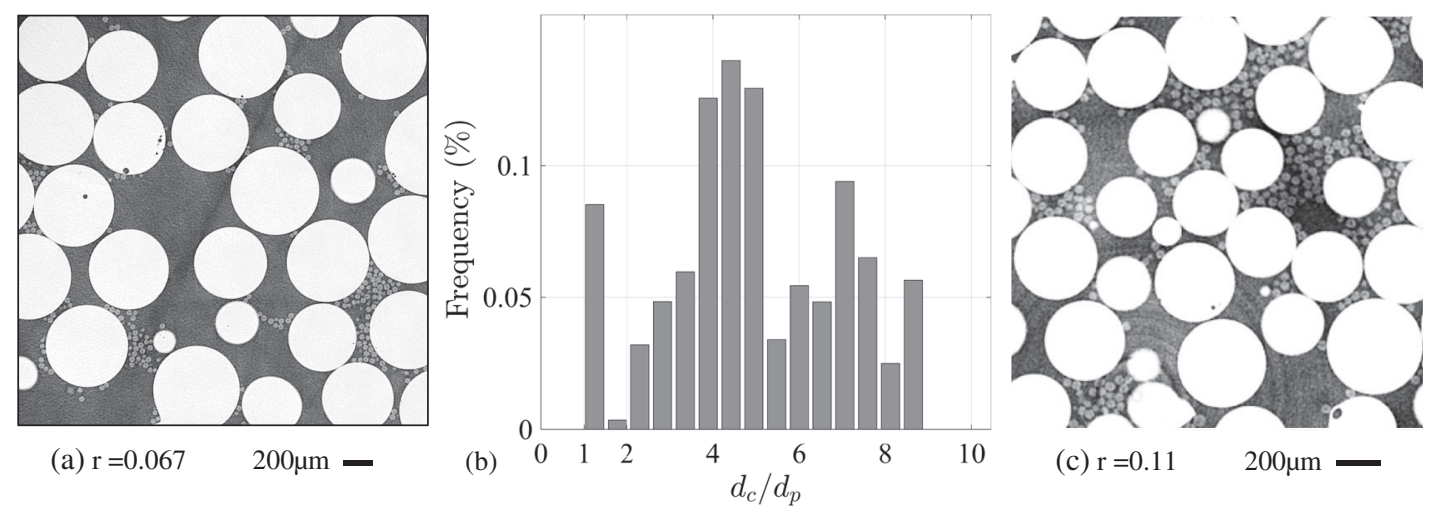

FIG. 3. View ( $\mu \mathrm{CT}$ image, $5 \mu \mathrm{m}$ voxels) of the internal structure after particle injection and drying at $x=4 d_{g}$ for $r=0.067$ (a) and $r=0.11$ (c). White regions correspond to grains, light gray to blocked particles, and dark gray to air. (b) shows an estimation of the size 
On average, this amounts to considering that a particle (in fact, a potential cluster) arriving at a free site in some layer has a specific probability to be blocked by size exclusion or otherwise it progresses to the next layer. Moreover, we assume that at each step of the process the flow rearranges to avoid filled sites, so that when they arrive at some layer the particles attempt to go through the free sites only (i.e., we neglect the flow through the clusters). Note that this is consistent with the fundamental assumption of previous numerical simulations [10], but our experiments show that this description become realistic only if it relies on the concept of a cluster containing a sufficient number of particles and blocking a large pore. This means that any new particle arriving in an already partially clogged layer will nevertheless have the same probability to meet a free site in this layer, the same probability to get stuck on it, finally some constant probability $p$ to get stuck in the layer, and a probability $1-p$ to progress to the next layer. Another way to think about this constant $p$ is that, while clogging goes on, the number of free sites in a layer decreases, but for the same global concentration of injected particles the number of particles arriving on the free sites increases reciprocally, thus keeping constant the probability of a clogging event in the layer. Note that the value of $p$ obviously depends on the way $e$ was defined.

As a result, the probability $f$ for a particle to get stuck in the layer $i+1$ is written as $f(i+1)=p\left[1-\sum_{1}^{i} f(j)\right]$. For $x \gg e$, a continuous version of this equation may be written as $e P(x)=\left[1-\int_{0<u<x} P(u) d u\right] p$, in which $P$ is the probability density function to get stuck at some position. We will assume that the solution of this equation, i.e., $P(x)=(p / e) \exp (-p x / e)$, constitutes a good approximation of the clogging process in our case. Note that $\lambda=e / p$ is an intrinsic characteristic penetration length, i.e., a function of the porous structure and particle to grain size ratio. It is worth emphasizing that the final model expression is similar to the basic conceptual model proposed for colloid transport [17] and for filtration [18], where $\lambda^{-1}$ was named the filter coefficient. However, despite its simple final form, our model, built on assumptions on the flow behavior derived from direct observations, takes into account both pore clogging and flow path evolution. Thus, it appears to be valid up to large cluster concentrations for which the significant part of the medium is clogged (and not only for a negligible particle number).

Since this distribution is valid for any injected particle no matter the clogging stage of the sample, the density of deposited particles in the system simply derives from the sum of $P(x)$ over the number of injected particles $N_{i}$ :

$$
n=\frac{p N_{i}}{N} \exp \left(-\frac{p x}{e}\right)
$$

The maximum value $n_{c}$ of $n$ at percolation (i.e., 0.45), i.e., that reached $x=0$ just before caking starts, is associated with the maximum number of particles that can be injected in the system, i.e., $N_{c}=n_{c} N / p$. Thus, the number of injected particles increases when $p$ decreases and tends to infinity when $p \rightarrow 0$, since the penetration length tends to infinity.

From a 3D representation of this solution [see Fig. 4(a)], we see that, for any $x$ and any $p, n$ increases linearly with $t=N_{i} / N_{c}$, which is in agreement with regime (1) of our experiments [see Figs. 4(b) and 2, inset]. The final distribution of particles in the sample (for $t=1$ ) shows that, for higher $p$ values, the particles tend to accumulate closer to the surface, as observed in our tests, and the critical injection number is reached sooner. Finally, the model predicts that for a given sample length, $N_{c}$ tends to its maximum possible value for $r \rightarrow 0$. This means, in agreement with the observed trends, that very low values of $r$ allow the best filling of a sample, in the limit of infinite times.

Let us now compare model predictions with the NMR results. We fit the model to our data by adjusting $p$ so that, for a given system (fixed $r$ value), all the experimental $n$
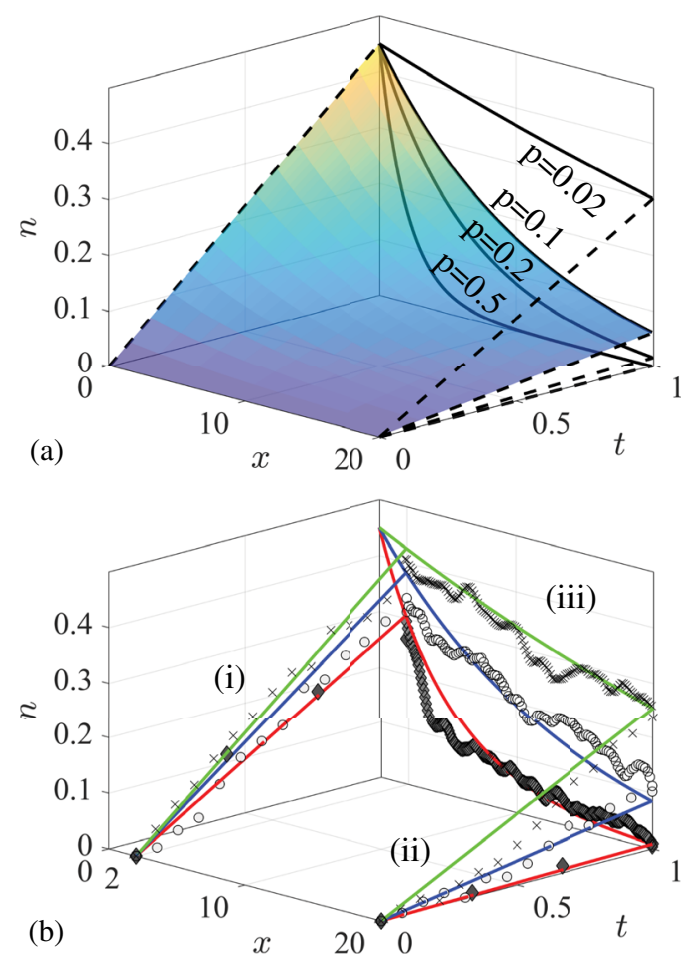

FIG. 4. Density of blocked particles as a function of the depth and injected particles. (a) Model predictions: surface mapping (colored) for $p=0.1$, final distributions before caking for different $p$ values (continuous lines), and time evolution at two specific depths (dashed lines). (b) Experimental data: time evolution at $x=2 d_{g}$ (i) and $x=20 d_{g}$ (ii), and final distribution (i.e., at $t=1$ ) (iii), for $r=0.13$ (filled diamonds), $r=0.11$ (circles), and $r=0.093$ (crosses). The continuous lines correspond to model predictions after fitting to all data for a given $r$ (see the text). 
profiles in time fall along the theoretical surface mapping. A series of profiles at extreme depths and time illustrate the agreement of the model with the data [see Fig. 4(b)]. As expected, the corresponding $p$ value increases with $r$ and tends to 1 (i.e., immediate blocking) for a value $\left(r_{c}=0.153\right)$ close to that corresponding to the typical maximum void size $\left(\approx d_{g} / 6\right)$ in granular packings. Finally, $p(r)$ may be well represented by the function $p=\exp (r-$ $\left.r_{c}\right) / \alpha$ (see Fig. S5 in Ref. [14]), with $\alpha=0.016$. Following the standard approach of capture by a spherical collector [18], we would expect a variation with $r^{2}$. The difference with our result is likely due to the flow distribution in these different paths, i.e., the fact that the local flow rate through small paths is smaller than elsewhere. We can finally note that the critical number of particles that can be injected essentially varies as $\exp -\left(r-r_{c}\right) / \alpha$.

The resulting relationship between $r$ and $p$ provides a means to adapt filter characteristics (pore size and thickness) to get expected filtration properties (e.g., distance of deposition). These results might serve to refine the numerical simulation of permeability evolution [10]. For example, they suggest that, for a homogeneous porous medium with realistic pore size distribution, there always exist, close to any large pore, some small pores which can be the source of clogging through the formation of a large "cluster" finally equal to the large pore. The principles of this model may be extended to clogging in porous media under more complex conditions, by considering that the different possible addi2 tional effects essentially affect the clogging probability value.

[1] C. Tien, Granular Filtration of Aerosols and Hydrosols (Butterworths, Boston, 1989); L. J. Zeman and A. L. Zydney, Microfiltration and Ultrafiltration: Principles and Applications (Marcel Dekker, New York, 1996).

[2] M. B. Rothberg, Circ.: Cardiovasc. Outcomes 6, 129 (2013); I. Vermes, E. T. Steinmetz, L. J. J. M. Zeyen, and E. A. van der Veen, Diabetologia 30, 434 (1987).

[3] Y. H. Faure et al., Geotextiles Geomembranes 24, 11 (2006).

[4] J. E. Altoe, P. Bedrikovetsky, A. G. Siqueira, A. L. S. de Souza, and F. S. Shecaira, J. Pet. Sci. Eng. 51, 68 (2006).

[5] J. A. C. Barth et al., Agron. Sustainable Dev. 29, 161 (2009); S. A. Bradford and M. Bettahar, J. Environ. Qual. 34, 469 (2005).
[6] Filtration and Drainage in Geotechnical/Geoenvironmental Engineering, edited by L. N. Reddi and M. V. S. Bonala, ASCE Special Publication (American Society of Civil Engineers, Reston, VA, 1998).

[7] J. Linkhorst, T. Beckmann, D. Go, A. J. Kuehne, and M. Wessling, Sci. Rep. 6, 22376 (2016).

[8] N. Roussel, T. L. H. Nguyen, and P. Coussot, Phys. Rev. Lett. 98, 114502 (2007); B. Dersoir, M. R. de Saint Vincent, M. Abrarian, and H. Tabuteau, Microfluid. Nanofluid. 19, 953 (2015); S. Massenburg, E. Amstad, and 5 D. A. Weitz; 20, 94 (2016); , 74, 061402 (2006); , 13, 37 (2017).

[9] S. Datta and S. Redner, Int. J. Mod. Phys. C 09, 15356342 (1998); Phys. Rev. E 58, R1203 (1998); , Water Resour. Res. 7343 42, W12S02 (2006).

[10] A. O. Imdakm and M. Sahimi, Phys. Rev. A 36, 5304 (1987); M. Sahimi and A. O. Imdakm, Phys. Rev. Lett. 66, 1169 (1991).

[11] G. Keir, V. Jegatheesan, and S. Vigneswaran, in Water and Wastewater Treatment Technologies, edited by S. Vigneswaran (Encyclopedia of Life Support Systems, Oxford, 2009).

[12] S. Faber, A. Al-Maktoumi, A. Kacimov, H. Al-Busaidi, S. Al-Ismaily, and M. Al-Belushi, Arab. J. Geosci. 9, 293 (2016).

[13] T. Amitay-Rosen, A. Cortis, and B. Berkowitz, Environ. Sci. Technol. 39, 7208 (2005); E. O. Fridjonsson, S. L. Codd, and J. D. Seymour, Transp. Porous Media 103, 117 (2014); A. P. Lehoux, S. Rodts, P. Faure, E. Michel, D. CourtierMurias, and P. Coussot, Phys. Rev. E 94, 053107 (2016); 9 A. P. Lehoux, P. Faure, E. Michel, D. Courtier-Murias, S. Rodts, and P. Coussot, Transp. Porous Media 119, 403 (2017).

[14] See Supplemental Material at http://link.aps.org/ supplemental/10.1103/PhysRevLett.000.000000 for particle suspensions, porous media, NMR procedures, injection cycles and automation, NMR procedure validation, critical saturation, $\mu \mathrm{CT}$ procedures, critical density value, and variations of parameter $p$.

[15] D. L. Huston and J. F. Fox, J. Hydraul. Eng. 141, 04015015 (2015).

[16] S. Torquato, Random Heterogeneous Materials (Springer, Berlin, 2002).

[17] K. J. Ives, Water Res. 4, 201 (1970).

[18] R. F Probstein, Physicochemical Hydrodynamics: An Introduction, 2nd ed. (Wiley, New York, 2003). 